\title{
Editorial Note: HCI Systems for Big-Data Based Multimedia Applications
}

(C) Springer Science+Business Media, LLC, part of Springer Nature 2017

Multimedia Tools and Applications gratefully acknowledges the editorial work of Prof. WenHsiang Hsieh on the special issue entitled, "HCI Systems for Big-Data Based Multimedia Applications."

This was an invitation-only special issue. Of the 35 papers invited to this issue, 19 were eventually accepted after two rounds of peer-review. 